\title{
NUEVOS HORIZONTES ACADÉMICOS PARA LOS ESTUDIOS DE TECNOLOGÍAS DE LA INFORMACIÓN Y LAS COMUNICACIONES EN ECUADOR
}

\author{
NEW ACADEMIC HORIZONS FOR THE STUDIES OF INFORMATION \\ AND COMMUNICATION TECHNOLOGIES IN ECUADOR
}

José María Díaz Nafría, Ph.D

Universidad Estatal Península de Santa Elena, Ecuador

Munich University of Applied Sciences

Facultad de Educación, Universidad de León, España

jdian@unileon.es

\author{
Washington Torres Guin, Ing. \\ Universidad Estatal Península de Santa Elena, Ecuador \\ Walter Orozco Iguasnia, Ing. \\ Universidad Estatal Península de Santa Elena, Ecuador
}

\section{RESUMEN}

Con objeto de redefinir el horizonte académico de las tecnologías de la información y las comunicaciones (TIC), de modo que los profesionales y el desarrollo de tales tecnologías estén a la altura de los problemas actuales del Ecuador se acomete en primer lugar una revisión crítica de la evolución histórica de tales horizontes. Como se observa en este artículo, la vertebración de los estudios en este campo de conocimiento ha estado marcado por una clara orientación positivista e instrumental inadecuada para lograr una realización efectiva del principio constitucional del buen vivir o Sumak Kawsay. La naturaleza de estas tecnologías hace que los problemas que deben tenerse en cuenta sean de corte global, pero no obstante existen retos, oportunidades, debilidades y fortalezas específicamente Ecuatorianas que corresponde tener en cuenta. Se plantea así una reconfiguración de los saberes que competen a las TIC basado en una integración de saberes que combina metodologías interdisciplinares y transdisciplinares para lograr soluciones innovadoras, responsables y sostenibles. Con estas premisas, se propone una re-elaboración crítica del curriculum a la luz del conocimiento pedagógico actual (en particular el aportado por el connectivismo, el neuroaprendizaje y, sobre todo, la pedagogía crítica).

Palabras clave: TIC, pedagogía crítica, conectivismo, neuroaprendizaje, interdisciplinariedad, innovación

\begin{abstract}
Aiming at re-shaping the academic horizon of the Information and Communication Technologies (ICT), so that professionals and the correspondingtechnologicaldevelopment come up to the current Ecuadorian problems, we first undertake the critical review of the historical evolution of such horizon. As it will be shown, the structuring of the studies in this knowledge field has been characterized by a clearly positivistic and instrumental orientation which is inadequate to achieve an effective realization of the constitutional principle of good-living or SumakKawsay. The nature of these technologies makes that the problems to be concerned of are global, but nevertheless there are challenges, opportunities, specificaIly Ecuadorian we have to consider. A restructuring of the knowledge related to ICTs is thus proposed, based on an integration of knowledge which combines interdisciplinary and transdisciplinary methodologies targeting innovative, responsible and sustainable solutions. On this basis, a critical reworking of the curriculum in the light of the current pedagogical knowledge (particularly the one provided by connectionism, neurolearning and, above all, critical pedagogy.
\end{abstract}

Keywords: TIC, critical pedagogy, connectivism, neurolearning, interdisciplinarity, innovation 


\section{Introducción}

Con objeto de poder hacer un análisis auténticamente crítico del horizonte epistemológico frente al cual se encuentran hoy en día las profesiones de ingeniero en electrónica y telecomunicación, así como la de ingeniero en informática (que son las que más directamente competen al campo de las tecnologías de la información y las comunicaciones, TIC), es necesario observar cuál ha sido la trayectoria epistemológica en la que se han conformado, históricamente, estas profesiones desde sus orígenes, que pueden cifrarse a mediados del siglo XIX. De esta manera, no contemplamos simplemente horizontes de saberes confiables, sino que distinguiendo de dónde venimos podremos valorar mejor hacia dónde conviene poner rumbo para la fundamentación epistemológica de unas de las profesiones que son de trascendental importancia en la era de la información para que sirvan a la empresa colectiva de construcción del Buen Vivir en Ecuador, lo cual implica atender a cada uno de sus variados contextos socio-culturales y socio-económicos.

\section{Escorzo histórico-crítico de los saberes que competen a las TIC}

Es a mediados del siglo XIX cuando, por una parte, las tecnologías de la comunicación se ven plenamente asistidas por el crecimiento de los saberes electromagnéticos $\mathrm{y}$, por otra, cuando las tecnologías informáticas se encuentran en status nascendi gracias a la organización progresivamente automatizada de procesos industriales, económicos y administrativos, así como por la fundamentación de las herramientas lógicas y formales que constituirán la base de las tecnologías de la computación electrónica en el siglo XX.

\section{Dimensión utópica e instrumental de la profesión}

Bastaría echar la vista a los años precedentes para comprender el tipo de recepción social que se le otorgaba a estos avances y que fraguaron el status atribuido a su fundamentación epistémica. Así, observamos como Vandermonde-desde su cátedra de economía política- consideraba que las virtudes técnicas del telégrafo bastaban "para hacer posible el establecimiento de la democracia en un gran pueblo." ¡Y eso en 1795, cuándo el telégrafo se encontraba técnicamente al nivel del usado en el imperio romano!1,2. Desde esta óptica cabe entenderse que la inmediatez otorgada por los medios eléctricos solo cabía recibirse con mayor entusiasmo. Este es el marco en el que la influyente escuela positivista de Chevalier reemplazará los seculares horizontes de utopía social por los de una utopía comunicativa que por sí misma desencadenaría todas las aspiraciones emancipadoras: "Mejorar las comunicaciones, es trabajar por la libertad real, positiva, práctica; es hacer que todos los miembros de la familia humana participen en la posibilidad de recorrer y explotar el globo que le ha sido dado en patrimonio [...]. Es hacer igualdad y democracia" ${ }^{3}$. Si se tiene en cuenta que éste es el substrato sobre el cual florecieron la mayor parte de las disciplinas científicas en las que se basa las profesiones vinculadas a las TIC, así como su matriz institucional, resulta del todo normal que cualquiera de sus avances se considerase per se en relación de causalidad con el progreso, y que se tomara como incuestionable la fundamentación epistémica que lograba el prodigio de la instantaneidad de las comunicaciones eléctricas, o el de la exactitud e inmediatez de cálculo de operaciones manualmente inabordables.

Se veía en estas virtualidades, por una parte, la solución a las limitaciones estructurales del mundo antiguo respecto a la participación de sujetos históricos emergentes y a la diseminación de conocimientos. Tanto la electricidad, como luego la electrónica, serían a su vez vistas como la condición de posibilidad de la descentralización (por ejemplo, así fue planteado por Kropotkin y Mumford, respectivamente), que completaría otra de las seculares aspiraciones emancipadoras1. No obstante, la propia historia de los proyectos de desarrollo de las tecnologías de la información y las comunicaciones, muestra que los sistemas de telecomunicación desarrollados fueron conformando el esqueleto organizativo de los procesos sociales de globalización económica y a escala más limitada, los de cualquier sistema socio-económico desplegado en un territorio extenso. Su relación, con el desarrollo de las redes financieras, de las redes comerciales, de las corporaciones multinacionales ha sido tan estrecha, que unas resultan inconcebibles sin las otras y socioeconómicamente, ha conducido a relaciones de dominación así como a una dosis desproporcionada de desigualdad de dimensiones múltiples ${ }^{4,5}$. Pero además, su vinculación al poder militar ha sido igualmente protagonista en determinados momentos y sectores clave que, de hecho, han sido decisivos para la construcción del corpus de conocimientos básicos para las profesiones en cuestión (piénsese en los trabajos de Shannon en comunicaciones bélicas, los de Turing en criptografía, o los de Wiener en el guiado de misiles; trabajos que en los tres casos estuvieron directamente implicados en la formulación de teorías decisivas para la telecomunicación, la computación y la automática). Observamos, por tanto, una instrumentalización 
de la profesión que la ha apartado del proceso de creación socio-cultural vinculado a utopías sociales (como cabe entenderse el Plan Nacional del Buen Vivir (PNBV) a la hora de articular el principio del buen vivir o Sumak Kawsay recogido en la Constitución ecuatoriana de 2008, § 275, 2786), en beneficio de intereses particulares. Dicha capacidad de instrumentación no concierne tanto a la validez del conocimiento adquirido como a su alejamiento de una fundamentación genuinamente crítica de la misma en el sentido horkheimeriano, y se vincula muy directamente alas bases institucionales del sistema científico-técnico asociado $^{7,8}$

\section{Del dominio de la epistemología positivista hacia una epistemología crítica}

En efecto, fue dentro del contexto positivista antes aludido en el que se crearon las bases institucionales de las profesiones concernidas, tanto en lo que atañe a su base epistemológica como a su desenvolvimiento práctico. El principio orgánico de las escuelas politécnicas superiores francesas -que a su vez constituyeron el paradigma para la institucionalización de la ingeniería en tantos otros horizontes- se fundaba en el principio positivista de irrestricta divisibilidad del trabajo científico. Dicha división había de sustentarse mediante la garantía de aplicación de una metodología científica que, gracias a un proceso de cautelosa reducción, solo daría pasos firmes, "claros, distintos" (por usar las categorías cartesianas e ilustradas de orientación científica), positivos, agregables al resto de los saberes conformados bajo la misma cautela y disciplina.

Este principio reduccionista que guía la metodología científica desde Descartes y que supone la analiticidad última de la realidad es bifaz: por una parte, la complejidad de la realidad atendida queda limitada por los instrumentos de control de laboratorio que la descontextualiza, eliminando los componentes relacionales con el entorno que también la constituyen; pero por otra, ofrecen al científico una soberanía que lo independiza de su contexto social y lo convierte en autor incontestable de verdades, cuyo rigor queda vedado al lego, aunque eso sí, bajo la vigilancia de sus pares, en virtud de un principio de reproducibilidad que a su vez se sustenta sobre un precepto de simetría explicativa ${ }^{9}$. Dicho sea de paso, esta supuesta simetría entre retrodicción y predicción así como las otras condiciones sobre las que se apoya la reproducibilidad empírica son, en última instancias, refractarias a la aprehensión de la complejidad de lo real; pero, no obstante, supone el único coto de "crítica" que admite la metodología positivista. De este modo, la complejidad propia de la realidad en la que están insertas los problemas del hombre queda reducida a la de un objeto acotado, segregado del todo al que pertenece. En definitiva,dicha complejidad queda distanciada de un legislador científico, que con su trabajo de laboratorio va conformando las riendas de dominio sobre esa parcela de la realidad sobre la que descarga su exclusiva atención, y que así queda a merced de los objetivos de quienes hacen uso de ella.

Tanto Descartes como Bacon, plantean muy directamente este principio de instrumentalización, pero lo conciben en beneficio del hombre en general. El proyecto de la ilustración -en su dimensión emancipadora- supondría que todos los hombres se encontrarán en dicha situación. Sin embargo, desde el siglo XVIII se ha constatado un crecimiento ininterrumpido de la desigualdad global que hace que el acceso a dichos beneficios sea tremendamente desigual y a la postre resulte un mecanismo de explotación del hombre por el hombre ${ }^{10,11}$. En el ámbito de las tecnologías que nos compete este problema hoy está agudamente representado -entre otros- por los proyectos de Big Data, que crean una diferencia sustantiva en el aprovechamiento de la información global por parte de corporaciones transnacionales, estados e individuos, y que de no reconducirlo podría suponer, en la era de la información, un horizonte de aún mayores desigualdades (como muestra la preocupación manifestada tanto en la opinión 4/2015 de la European Data Protection Supervision de la Unión Europea ${ }^{12}$, así como la encíclica papal 'Laudatio si' ${ }^{13}$. Este es un ejemplo de problemas que urge abordar desde una perspectiva epistemológica crítica. Para salvar este escollo, la pedagogía crítica ofrece un ámbito de aprendizaje para la formación de dicha base epistemológica en las profesiones implicadas que supondría una ruptura respecto a una matriz positivista que -como se justificó anteriormente- cercena de raíz la posibilidad de orientación del proyecto científico-técnico hacia un rumbo como el marcado por el PNBV ${ }^{14}$.

Resulta curioso que esta matriz institucional de corte positivista y su correspondiente fundamentación epistémica, hayan llegado hasta nuestros días a pesar de la acumulación de evidencias científicas acerca de su radical insuficiencia y error. $Y$ resulta, particularmente llamativo, dentro del horizonte epistemológico de nuestra profesión por tres magnos expedientes: (i) en la medida que el avance de la teoría electromagnética -que sigue constituyendo un núcleo teórico central- llevó a la ruptura con la física clásica a través de la teoría de la relatividad;(ii) en cuanto a que creación de la teoría cuántica -sobre la cual se fundamen- 
tan la mayor parte de los progresos en electrónica- puso en radical entredicho la analiticidad de lo real; y (iii) debido a que la fundamentación lógica de la matemática -que condujo tanto a la informática como al principio de indecibilidad de Gödel- desnuda la aporía del positivismo en sus fundamentos últimos. La ausencia curricular de fundamentos teóricos en relatividad, en cuántica, en lógica y matemática que podemos encontrar por doquier en la enseñanza de las profesiones concernidas supone un bloqueo adicional de la dimensión crítica que nos gustaría construir. Por el contrario, agregar estos componentes al horizonte epistemológico de la profesión ofrecería una "opción de rejuvenecimiento espiritual, de mutación brusca que contradice el pasado para reconfigurar el presente" 14 .

\section{De la era industrial a la era de la in- formación}

Pero para analizar los cambios de rumbo en el horizonte epistemológico, es necesario prestar atención a un cambio histórico operado a nivel social y que está impulsando transformaciones radicales en el mundo científico-técnico y muy especialmente, en nuestras profesiones. Nos referimos a lo que suele referirse como cambio de era: de la industrial a la informacional. En efecto, en la era industrial la "fábrica" presenta una dinámica de cambio relativamente lenta, aunque superior a la de la etapa preindustrial $-y$ en ocasiones incluso más lenta que en aquella-.

Su virtualidad radica en la intensificación de los procesos, que se vincula tanto a la efectividad del aprovechamiento de energía en el proceso de producción como en la disponibilidad de energía para intensificar dicho proceso (hasta el punto de que el uso de energía por habitante se convierte en cuantificador del progreso ${ }^{15}$ ). Bajo este prisma, el perfeccionamiento de los procesos técnico-productivos constituye la clave de bóveda del progreso técnico en la etapa industrial. Esto requiere planificación, modelado de las herramientas, de los instrumentos y de los sistemas industriales. En el caso del despliegue de las tecnologías de comunicación el problema tiene un carácter adicional: mientras la intensificación de la producción industrial se encuentra en relación directa con el aumento de usuarios, en el caso de la telecomunicación (salvo en el caso de estructuras de difusión), la complejidad e intensificación de elementos del sistema de comunicación se encuentra en relación cuadrática con el número de usuarios, debido al número de enlaces necesarios $^{16}$. Por esta razón, la necesidad de investigación científica se encontraba estructuralmente ligada al principio de expansión del propio siste- ma, que de otro modo hubiera sido inviable. Los descubrimientos en electrónica, en física de estado sólido, en dispositivos ópticos, en conmutación digital... constituyen pasos con los que dicha expansión fue llevándose a cabo. En cualquier caso, la planificación de los sistemas industriales y de telecomunicación respondía a un patrón dinámico de relativa estabilidad en lo que concierne al mundo de la vida. La fiabilidad de las máquinas y del suministro de telecomunicaciones suponían una constante sobre la que organizar el resto de procesos socio-económicos.

Sin embargo, esto empezó a cambiar significativamente en los años 70 del siglo $\mathrm{XX}$, debido a múltiples factores: el coste de las fuentes de energía, la saturación de la producción en los países más industrializados, el poder creciente de oligopolios globales... pero además en lo concerniente a los avances en tecnologías de la información y la comunicación: la posibilidad de vertebrar los procesos económicos al margen de los estados-nación, la disponibilidad de medios automáticos para la intensificación de la producción y la consecuente posibilidad de reducir el peso relativo de las fuerzas laborales en las relaciones de producción (como advirtió el propio Norbert Wiener, padre de la cibernética y que en efecto se ha convertido en un problema de estancamiento social en los países más industrializados) $)^{17,4,5}$. En última instancia, se estaba produciendo un cambio de época, en la que lo central dejaba de ser la intensificación de los procesos industriales mediante una mayor y más eficiente disponibilidad energética, para dejar paso a la posibilidad de reconfigurar flexiblemente los procesos productivos a escala planetaria. Una reestructuración de dichos procesos en la era anterior implicaba la reorganización humana de los mismos o el rediseño y construcción de nuevas máquinas; en la era de la información basta con cambiar el programa de la máquina, en "informar" adecuadamente a la máquina: la dinámica de la organización de los procesos socio-económicos se acelera. La "información" de los procesos de producción confiere a estos una virtud de flexibilidad que contrasta con la virtud de intensificación (energética) de la producción característica de la era industrial. Pero para el mundo de la vida (tanto individual como colectiva) esta dinámica de flexibilidad de los procesos productivos y económicossupone un problema nuevo: hay que contar con una acelerada dinámica de cambio que exige una cultura adecuada, una ética nueva y de esta elaboración el profesional de las TIC no puede quedar al margen ${ }^{5}$.

En la década siguiente, la de 1980 , se asiste al desmantelamiento institucional de la organización científico-técnica y administrativa de las TIC 
que desde el Keynesianismo se había mantenido en manos de los estados. $Y$ se produce también una transformación en la estructura económica mundial con una fuerte financiarización y deslocalización de la misma que restringirán el dominio de actuación de los estados-nación, no obstante, legitimadores de las relaciones geo-económicas establecidas. El despliegue de las tecnologías de la información en una nueva gama de productos y servicios vino acompasado por un reverdecimiento de la confianza en las telecomunicaciones como palanca de cambio para todos los progresos imaginables, en un ciberutopismo cuyo tenor se encuentra llamativamente cerca al de Vandermonde o Chevalier antes mencionados ${ }^{19,1}$. Los proyectos de las "autopistas de la información" anunciados por la administración norteamericana tuvieron un efecto sobre el horizonte epistemológico de la profesión: los sistemas se sobredimensionan, lo cual tendrá en la década siguiente, tras la burbuja de las ".com", el efecto de que en los países más industrializados la planificación de los propios sistemas de telecomunicación dejara de encontrarse entre las prioridades técnicas. En su lugar se establece la exigencia de idear servicios para ocupar los recursos disponibles con rendimiento económico. Bajo el dominio del paradigma neo-liberal carece de sentido planificar la atención de necesidades sociales, solo hay consumidores y proveedores de servicios, el mercado desregulado se convierte paradójicamente en el único ente regulador, y se supone que su círculo virtuoso conduce -como en la teoría económica clásica- a una eficiente satisfacción de las necesidades. La realidad de las brechas digitales muestran una realidad muy distinta ${ }^{5,20}$.

\section{Hacia una nueva vertebración de los saberes profesionales a la altura del tiempo}

Una adecuada recomposición de los saberes que competen a las TIC debe buscar una nueva vertebración académica orientada a la formación de profesionales, capaces de dar la cara a los problemas actuales en sus diferentes dimensiones: global, nacional y local. El anterior bosquejo histórico-crítico ha destacado un conjunto de retos de carácter global que reviste peculiaridades regionales y locales para Ecuador, marcadas por el hecho de no haber disfrutado -como el resto de países de la región- de posiciones de dominio tecnológico global, esto es, de fuentes y medios de información y comunicaciones, así como de los saberes científico-técnicos que permitan el desarrollo de soluciones endógenas o de penetración en el mercado global. Por otra parte, el ni- vel general de desarrollo educativo y científico en Ecuador-que presenta ciertas similitudes regionales- requiere, pese a los avances registrados en los últimos años, de mejoras sustanciales para poder afrontar de un modo efectivo y endógeno los problemas que afrontan nuestras sociedades. En este sentido, es importante tener en cuenta que mientras la escasez de conocimiento especializado podría ser una dificultad a la hora de poder competir en el mercado global de productos y servicios de información y comunicaciones, la confrontación adecuada de los problemas complejos que acechan tanto a las sociedades más industrializadas, como a las que lo están menos, no dependen tanto -como se verá más adelante- del conocimiento especializado, sino de la capacidad de integrar conocimientos y puntos de vista. En esto, la desventaja de carecer de especializaciones dentro del área podría constituir una ventaja que debe aprovecharse para la configuración de un horizonte epistemológico más adecuado al desarrollo de las TIC a la altura del tiempo. Del mismo modo, los marcos político-regulatorios constituidos por la propia constitución, el PNBV, la Ley Orgánica de Telecomunicaciones, la Ley de Economía Popular y Solidaria, y la Ley de Economía Social del Conocimiento (actualmente en desarrollo), ofrecen espacios de búsqueda de soluciones inéditas a problemas que competen de lleno a las TIC.

\section{Recuperación de epistemologías integradoras}

Como consecuencia de una aproximación mercantilista a la prestación de servicios de información y comunicaciones, se pierde en buena medida la dimensión sistémica que había sido central en el desarrollo de las TIC en la era precedente (ausencia que de hecho puede encontrarse en las mallas curriculares vigentes en Ecuador). Sin orientación sistémica, el campo de la "innovación abierta", creado en la década pasada, promueve (tal y como es mayoritariamente concebida en EE.UU. y la U.E.) la conexión libre de actores tecno-científicos sin orientación alguna, y sin acuerdo ni fundamentación epistemológica sólida, para la creación de servicios de los que pretende extraerse beneficio a corto plazo, antes de que dichos procesos sean optimizados y planificados por trabajadores tecno-científicos con una buena base epistémica ${ }^{21}$. Así, presenciamos una relativa decadencia de los sistemas educativos (en cuanto a las competencias científicas que ofrecen) en países que apuestan por esta estrategia económica. Este es, por ejemplo, el caso de Suecia, que en el curso de una década ha pasado de uno de los mejores resultados educativos a situarse por debajo de la media de la educación europea a la 
vez que mantiene su competitividad económica en los niveles más altos de la Unión Europa (UE) ${ }^{22}$.

Tanto la innovación abierta (centrada en la economía de mercado) como la innovación social (cuyas metodologías y prácticas tienen un origen muy anterior y apuntan a objetivos más amplios ${ }^{23}$ ) ofrecen, sin duda, la posibilidad de poner en conexión puntos de vista diversos en la confrontación de los problemas sociales que atañen a las TIC (en particular aquellos que pudieran quedar desatendidos por las prácticas burocráticas en el seno de organizaciones públicas y privadas). Sin embargo, la carencia de una metodología que permita la consecución sistemática de soluciones y la ausencia de una epistemología que haga posible orientar la práctica, resta a estas estrategias valor científico y técnico. Se requeriría el recurso de vínculos más orgánicos a la hora de vehicular adecuadamente el momento que podemos llamar creativo hacia el momento técnico de búsqueda de soluciones y de construcción de conocimiento científico.

La perspectiva conectivista se centra en el primer momento, la de facilitar la interacción de actores en red para la creación de sentidos -cuya importancia es sin duda capital-,y da por garantizado el subsiguiente desarrollo del aprendizaje adecuado y la creación de conocimientos ${ }^{24}$. Sin embargo, del mismo modo que la red de interacciones moleculares puede ser capital para dar lugar a un organismo vivo (y la consecuente emergencia de propiedades inexistentes en los componentes químicos), el aprendizaje y la creación de conocimiento requiere de una adecuada articulación de sentidos para que puedan darse, esto es, epistemologías que hagan posible la integración del conocimiento.

Por otra parte, el nuevo escenario de la era de la información -que caracterizábamos antes y que en cuanto a uno de sus rasgos constituyentes supone el aumento de las interacciones entre agentes que se encuentran repartidos a lo largo y ancho del globo- plantea retos adicionales. Según ha sido estudiado recurrentemente y desde diversos enfoques, la complejidad de los problemas que confrontan nuestras sociedades crece a un ritmo que es superior al que pueden abordarse por medio de la ciencia disciplinar por mucho que intensifiquemos sus empeños ${ }^{25}$. Esto ha conducido a que instituciones internacionales como el European Research Area Board, la National Science Foundation, o la OCDE lleven varias décadas alertando acerca de la necesidad de confrontar los problemas de un modo interdisciplinar o transdisciplinar26,27,28. Según estas y otras instituciones, estos modos de investigación y conocimiento científico serían las únicas estrategias viables para confrontar de un modo adecuado la complejidad que nos acecha en el momento presente y que acumula grandes retos de orden ecológico, socio-económico, geo-político, inter-cultural e identitario. Sin embargo, las mismas instituciones constatan cómo la dinámica institucional de la organización académica especializada supone un escollo que frena cualquier avance en este sentido. En Ecuador, debiera aprovecharse la ausencia de esas fuertes tradiciones disciplinares para poder desarrollar tales enfoques que encuentran resistencias en el seno de las organizaciones científico-técnicas norteamericanas y europeas con el objeto de sortear el problema de reproducir una epistemología inapropiada.

La interdisciplinariedad exige una disposición de apertura, de saber trabajar con otras disciplinas mediante procesos de intercomprensión. El estudio de la información -que atraviesa casi todas las disciplinar científicas-, además de resultar central para la vertebración epistémica de las TIC, supone un ámbito que desde sus orígenes se ha desarrollado en ámbitos interdisciplinares (aunque en su multiplicidad estos hayan resultado ser generalmente inconexos). La interdisciplinariedad, que tanto en el estudio de la información como de la comunicación podrían ofrecer un mecanismo de interactuación de agentes científicos, técnicos y sociales muy apropiado para una contextualización adecuada del horizonte de saberes pertinentes, presenta a su vez un riesgo: el de la multiplicación de disciplinas que nacen en la convergencia de otras y que se constituyen como disciplinas con derecho propio ${ }^{29}$. Debiera, por tanto, promoverse un estudio interdisciplinar de la información y la comunicación que sea general, no restringido a una convergencia de disciplinas por mera conveniencia -con lo que se evitaría adicionalmente una reducción de la complejidad.

Esto, probablemente, implica que sería necesario participar en redes epistémicas que desbordan las facultades y las universidades, entrando en contacto con investigadores y dominios científicos que cubran todas los aspectos de la realidad en cuestión. Como sostiene el conectivismo, la formación de sentido en los procesos tanto de aprendizaje como de investigación, podrían verse significativamente favorecidos por esta estructura de interdisciplinariedad en red $^{24}$.

La transdisciplinariedad queda implicada por los enfoques generalistas de la ciencia de sistemas para el estudio general de la realidad y de la ciencia de diseño para el estudio de lo artificial (en sentido amplio) a los que antes hacíamos referencia. Su concurso en el horizonte epistemológi- 
co de la profesión, ofrece una plataforma para la creación de una visión holística de los problemas que competen a la profesión y de los agentes implicados. Como señala la Organización de Estandarización Internacional, este aspecto constituye la espina dorsal de la responsabilidad social de las organizaciones ${ }^{30}$.

Serían precisamente estas estrategias de integración científica las que podrían completar de un modo sistemático la formación de sentido a la que hacíamos alusión al hablar de la innovación social e innovación abierta. Con esta finalidad, los autores promueven desde el esfuerzo coordinado de una red internacional de instituciones académicas reunidas en torno a la iniciativa PRIMER (Promoting Interdisciplinary Methodologies in Educationan Research) la necesidad de complementar la creación de espacios híbridos de fomento de la creatividad tecno-científica (propio de la innovación abierta) con la ciencia de sistemas(dotada de una epistemología robusta) y con la ciencia de diseño, esta última muy apropiada para un desarrollo consciente y responsable de la tecnología en su contexto social y medioambiental ${ }^{31}$. Estos complementos a la innovación abierta, fundados tanto en los paradigmas de la teoría de sistemas y de la teoría de complejidad, ofrecen una aproximación más apropiada al encauzamiento y creación de conocimiento en los procesos de innovación.

En su conjunto, esta combinación permite el desarrollo de vínculos orgánicos entre los procesos creativos, el conocimiento científico, la investigación (a los que nos referíamos más arriba) y la vinculación con la sociedad (inherente a la innovación social). Según el planteamiento de Larrea $^{32}$, esto permitiría el desarrollo adecuado de las dimensiones curriculares en educación superior desde la complejidad sistémica, y a su vez, el cumplimiento de las "funciones sustantivas"de la educación superior en el contexto actual (esto es, formación, investigación, gestión del conocimiento y servicio social).

En el campo de las TIC, la ciencia de sistemas ofrece adicionalmente una perspectiva de gran valor para la articulación de saberes y prácticas: la gestión adaptativa de las organizaciones vertebrada por un sistema de información y comunicaciones de acuerdo al modelo de sistema viable ${ }^{33}$. La posibilidad de aplicación de este modelo a problemas de naturaleza política, social, científica y organizacional abre perspectivas de gran valor para el desarrollo integral de las mencionadas funciones sustantivas de la educación superior en el campo de las TIC y en conexión orgánica con el resto de disciplinas científicas ${ }^{5}$.

\section{Reelaboración crítica del currícu- lum desde el conectivismo, el neu- roaprendizaje y la pedagogía crítica}

Para una reforma curricular que tenga en cuenta los descubrimientos realizados por el conectivismo24, resulta capital -en el proceso de aprendizaje- favorecer desde un principio la formación de la red semántica en la que se desenvuelve el quehacer profesional, poniéndolo en conexión interdisciplinar con los otros campos del saber de los que pueda nutrirse el aprendizaje ulterior del estudiante.

Una articulación temprana de la correspondiente red semántica facilita un aprendizaje más autónomo, y por tanto, más auto-regulado. Virtudes que son igualmente destacadas por el neuroaprendizaje $^{35,36}$. La adquisición de términos clave que funcionen a modo de concentradores de red, resulta particularmente interesante. Para ello, resulta conveniente la formación de esos conceptos clave e hiper-conectados, cuanto antes en la medida que su adquisición ofrece la posibilidad de dar saltos cualitativos en la sucesiva adquisición de conocimientos y de la interrelación de saberes pertinentes ${ }^{37}$. Para ello, es importante el desarrollo de materias que proporcionen una introducción general a los conceptos, teorías y problemas más relevantes del ámbito profesional.

Desde el punto de vista del neuroaprendizaje, resulta además importante la creación de un contexto apropiado y motivador para el aprendizaje que no resulte ajeno a lo emotivo y motive desde las etapas más tempranas la autoregulación ${ }^{35,36 .}$ Se considera así, que una de las orientaciones generales de la reforma curricular debiera apuntar hacia la creación de una suerte de ecología de aprendizaje que favorezca la inmersión e implicación del estudiante por medio de proyectos interdisciplinares desde los que la integración y pertinencia de los saberes surjan de modo natural. De este modo, se suscita la comprensión espontánea de facetas importantes sin las cuales no es posible resolver grandes grupos de problemas, cuyo paradigma se encuentra en el desarrollo metódico de proyectos concretos que el estudiante ha de confrontar. Será tarea de los docentes el encontrar -en colaboración con los estudiantes y la comunidad- proyectos que puedan servir para cubrir de una forma amplia los saberes profesionales de pertinencia más medular. Medular en el sentido de que, a través de ellas, se puedan derivar otros saberes disciplinares (por ejemplo, del electromagnetismo puede derivarse las teorías de propagación, de antenas, de transmisión, etc.). En cuanto a la temática y objetivos de los proyectos, se considera crucial que estos ofrezcan un sen- 
tido de vinculación con los problemas reales de la comunidad y que requieran una aproximación de trabajo en equipo. De este modo, la combinación de una aproximación crítica a la pertinencia de los saberes y el resto de las características de contextualización del aprendizaje recrean adicionalmente los criterios de la pedagogía crítica14.

La combinación, por tanto, de estos criterios de reforma curricular -como hemos visto, acordes con el conectivismo, el neuroaprendizaje y la pedagogía crítica- permitirían el desarrollo de una formación crítica, holística, participativa y creativa. El punto de arranque del aprendizaje estaría constituido, como se indicaba antes, mediante introducciones críticas al horizonte histórico, socio-cultural, científico-técnico y organizativo de la profesión. De esta manera, el estudiante podría configurarse su propio mapa y rumbos de aprendizaje, sin la desorientación que actualmente caracteriza, predominantemente, al individuo de la sociedad de la información perdido en un océano de informaciones y datos -a menudo dudosas-, pero que también ha caracterizado previamente el aprendizaje tradicional de la profesión haciendo que su gobernalle quedara en manos del cuerpo docente.

\section{Reconfiguración de los saberes profesionales}

Esta vertebración del horizonte epistemológico curricular implica una reorganización del conjunto de los saberes, que son necesarios para el desempeño eficiente de la profesión y de las exigencias del proyecto colectivo del Buen-Vivir, con independencia que en el proceso diacrónico de aprendizaje, estos saberes vayan emanando del sucesivo descubrimiento de su pertinencia. A modo de ejemplo: si la solución de los problemas de comunicación en una sociedad compleja, como la ecuatoriana, implica la utilización de medios electromagnéticos que hagan posible y efectiva la comunicación a grandes distancias, resulta obligado conocer la naturaleza de dichos fenómenos; pero pronto observamos -incluso partiendo de conocimientos elementales- que éstos están regidos por unas ecuaciones diferenciales que requiere mayores conocimientos de las correspondientes disciplinas matemáticas y a su vez se vinculan a otros fenómenos físicos que reclaman su atención específica. Según este planteamiento, la orientación del docente es capital para proporcionar al estudiante las oportunas orientaciones teóricas y de dependencia disciplinar. Pero, además, corresponde al andamiaje institucional el que se establezcan vínculos orgánicos entre las diversas disciplinas científicas que permitan articular la interdisciplinaridad que es precisa para la confron- tación de los problemas reales en su integridad. Como se señalaba antes, esto implica contar con una pre-ordenación de las áreas de conocimiento inherentes a la profesión que habrán de quedar debidamente cubiertas por el cuerpo docente, no solo especializado en TIC, sino del resto de disciplinas que fueran necesarias.

Según los criterios antes mencionados, dichos saberes deberán integrar, no solo compontes disciplinares (como pueden ser las ecuaciones diferenciales, o el análisis lógico de enunciados) sino otros de carácter interdisciplinar para facilitar la conexión con otras áreas de conocimiento de eventual importancia para el abordaje de problemas complejos (que a continuación se marcan $\operatorname{con}^{*}$ ) y transdisciplinar o sistemático para la integración de saberes en la planificación eficiente de las soluciones técnicas (que se marcarán con+). Así, desde las materias introductorias a las que nos referíamos antes (de carácter histórico, crítico-social y científico-técnico) y de la confrontación a proyectos socio-técnicos (para una involucración temprana del estudiante), el resto de saberes irá emanando de la necesidad de afrontar la complejidad asociada. Entre los más específicos a las profesiones implicadas cabría citar: teoría de la señal; teoría de la información*; teoría de la comunicación*; electromagnetismo (general y aplicado -teoría de circuitos, campos, guiado de ondas, propagación, antenas, fotónica, compatibilidad electromagnética); teoría de la codificación (incluyendo la criptografía clásica y cuántica); electrónica (dispositivos, instrumentación, sistemas analógicos y digitales); nanotecnología;sistemas de telecomunicación+ (transmisión, conmutación, ecosistema de la información);teoría de la computación (incluida clásica, cuántica, natural); programación (sistemas operativos, estructurada, orientada a objetos, visual, web);estudio de redes (teoría general, redes computacionales, de comunicación, semánticas);organización y gestión de datos-información-conocimiento+(bases de datos, inteligencia artificial, sistemas expertos).

Por otra parte, y con objeto de afianzar la epistemología transdisciplinar, debieran realizarse una intensificación de los contenidos en herramientas matemáticas, fundamentación lógico-matemática, modelización y simulación de sistemas, en física teórica, así como los estudios generales de: sistemas+ (incluyendo cibernética, automática, organización), complejidad+ (incluyendo teoría de redes, caos), diseño+ (incluida la interacción hombre-máquina; diseño medio-ambiental; ética de la ingeniería).

Finalmente, para sustentar la componente crítica y de vinculación con la sociedad se requeriría de complementos como: sociología de la comunica- 
ción, historia de las TIC, economía política y administrativa de las TIC, antropología, comunicación y cooperación intercultural.

Es evidente que el conjunto de saberes implicados desborda a una facultad dedicada a las TIC $y$, en esa medida, resulta vital un adecuado entramado institucional.

\section{Conclusión}

En conclusión, una reorientación del horizonte epistemológico para las carreras que competen a las TIC, de cara a contribuir de un modo eficiente al PNBV en nuestro contexto socio-cultural concreto, implica: la erección de una aproximación sistémica a los problemas concernidos sin merma de su complejidad y que habilite la conexión con otros dominios científicos, técnicos y sociales para la resolución de problemas de un modo conjunto; el desarrollo de un escenario epistemológico crítico en el que el conocimiento y las soluciones técnicas se entiendan en su dinámica dialéctica, es decir, en un continuo estado nascendi que exige creatividad y apertura de miras, imaginación y colaboración; y finalmente, una disposición al trabajo interdisciplinar que esté abierto a la intercomprensión activa de otros agentes científicos, técnicos y sociales en la medida que estos son capaces de aportar perspectivas epistémicas necesarias para confrontar la multidimensionalidad de los problemas complejos desde nuestro contexto socio-cultural concreto. Estar a la altura de los retos de la sociedad de la información y del conocimiento, requerirá -sin duda- del concurso de las tecnologías concernidas y de profesionales que sean capaces de encontrar soluciones adecuadas en una colaboración activa, responsable, consciente, crítica y creativa con el resto de agentes científicos y sociales.

\section{Referencias Bibliográficas}

1. Mattelart, A. Historia de la Sociedad de la Información. Barcelona: Paidós; 2007.

2. Aschoff, V. Geschichte der Nachrichtentechnik. Band 1: Beiträge zur Geschichte der Nachrichtentechnik von ihren Anfängen bis zum Ende des 18. Jahrhunderts. Berlin: Springer Verlag; 1989.

3. Chevalier, M. Lettres sur l'Amérique du Nord. París: Librairie C. Gosselin; 1837.

4. Díaz Nafría, J.M. The need for a systematic systems approach to security. TripleC. 2011; 9(1): 93-121.

5. Díaz Nafría, J.M. Ethics at the age of informa- tion. Systema. 2014; 2(1): 43-52.

6. AsambleaNacional. Constituciónde la República del Ecuador. Quito: Asamblea Constuyente; 2008 [Accesible en: http://www.asambleanacional.gob.ec/]

7. Horkheimer, M. Teoría tradicional y teoría crítica. Barcelona: Paidós; 2000.

8. Chomsky, N. Los Mercados y la "sustancia de la sociedad”. En J.E: García-Albea, N. Catalá, J. A. Díez Calzada (coords.), Los límites de la Globalización, Barcelona: Ariel; 2002.

9. Suppe, F. (ed.). Structure of Scientific Theories, 2nd edn. Urbana:University of Illinois Press; 1977.

10. Milanovic, B. Global Inequality and the Global Inequality Extraction Ratio. The Story of the Past Two Centuries. Policy Research Working Paper 5044. World Bank - Development Research Group; 2009.

11. Jolly, R. Inequality in Historical Perspective. ResearchPaper No. 2006/32. Helsinki: World Institute for Development Economic Research, United Nations University; 2006.

12. European Data ProtectionSupervision - Unión Europea. Towards a new digital ethics - Data, dignity and technology. Opinion 4/2015 [Internet]. Bruselas: European Data ProtectionSupervision - Unión Europea; 2005 [Consultado en junio de 2016] Accesible en: https://secure.edps.europa. eu

13. Francisco. Carta Encíclica Laudato si', n.3. [Internet]. Vaticano; 2015 [Consultado en junio de 2016] Accesible en: http://w2.vatican.va/content/ francesco/es/encyclicals

14. Ramirez Bravo, R. La pedagogía crítica. Una manera ética de generar procesos educativos. Folios. 2008; 28, 108-119.

15. Harris, M. Antropología cultural. Madrid: Alianza; 1995.

16. Pierce, J.R. Signals. The Science of Telecommunications. London, UK: W H Freeman \& Co.; 1990.

17. Noble, D.F. Progress Without People: New Technology, Unemployment, and the Message of Resistance. Toronto: Between the Lines; 1995.

18. Noble, D.F. Forces of Production. A Social History of Industrial Automation. New Brunswick, New Jersey: Transaction Publisher; 2011. 
19. Díaz Nafría, J.M. Telecomunicación e historia.Le Monde Diplomatique ed. Española. 2007; 10(35): 30. [Accesibleen: https://bitrum.wordpress.com/]

20. Warschauer, M. Technology and Social Inclusion: Rethinking the Digital Divide. Cambridge, Mass.: Massachusetts Institute of Technology; 2004.

21. Chesbrough, H. W. Open Innovation. The New Imperative for Creating and Profiting from Technology. Boston: Harvard Business SchoolPress; 2003.

22. Comisión Europea - CE. Europe 2020 in Sweden [Internet]. Bruselas: Comisión Europea [consultado en agosto 2014] http://ec.europa.eu/europe2020/europe-2020-in-your-country/sverige/ country-specific-recommendations

23. Mumford, M.D. Social Innovation: Ten Cases from Benjamin Franklin. Creativity Research Journal. 2002; 14(2), 253-266.

24. Siemens, G. Connectivism: A learning theory for the digital age. International journal of instructional technology and distance learning. 2005; 2(1).

25. Homer-Dixon, T.F. El vacío de ingenio. Madrid: Espasa Libros; 2003.

26. European Research Advisory Board - EURAB. Interdisciplinarity in Research. Final Report; 2004 [Internet]. Bruselas: European Commission - European Research Advisory Board (EURAB) [consultadoenagosto 2014]http://ec.europa11.eu/ research/eurab

27. European Research Advisory Board - EURAB (2012). The new Renaissance: will it happen? Innovating Europe out of the crisis. Third and Final Report of the European Research Area Board. Brussels: European Commission - European Research Area Board, [consultadoen Agosto 2014] Accesibleen: http://ec.europa.eu/research/erab/ pdf/3rd-erab-fina-report_en.pdf

28. National Academy of Science - NAS. Facilitating Interdisciplinary Research. National Academy of Sciences (NAS), National Academy of Engineering (NAE) and Institute of Medicine (IM). Washington: The National Academies Press; 2005.

29. Marijuán, P. El avance de la ciencia de la información. ¿Es necesario un nuevo modo de pensar?. En J.M. Díaz y F. Salto (eds), ¿Qué es información? León, España: Universidad de León;
2008. p. 523-534.

30. International Standard Organisation - ISO. Guidance on social responsibility. ISO 26000:2010. Geneva, Switzerland: International Standard Organisation; 2010.

31. Primer.unileon.es [Internet]. León, España: PRIMER: PRomotingInterdisciplinaryMethodologies in Education and Research. [consultada en Mayo de 2016] http://primer.unileon.es

32. Larrea de Granados, E. El currículo de la educación superior desde la complejidad sistémica. [Internet] Quito: Consejo de Educación Superior - CES; 2014 [consultado en junio de 2016] www. ces.gob.ec

33. Beer, S. Brain of the Firm. 2nd ed. London, UK: Wiley; 1981.

34. Díaz Nafría, J.M. eSubsidiarity: An Ethical Approach for living in complexity.En W. Hofkirchner, M. Burgin (eds.), The Future Information Society: Social and Technological Problems. Singapour: WorldScientific Publishing; 2016. [en prensa]

35. Lee, H.W., \& Juan, C.H. What can cognitive neuroscience do to enhance our understanding of education and learning?Journal of Neuroscience and Neuroengineering. 2013; 2(4), 393-399.

36. Organisation for Economic Co-operation and Development - OECD. Understanding the Brain: The Birth of a Learning Science. OECD; 2007.

37. Solé, R.V.; Corominas-Murtra, B.; Fortuny, J. Lenguaje, redes y evolución. Investigación y ciencia. 2003; 40, 58-67. 